\title{
Aqueous Extract of Saffron (Crocus sativus) Increases Brain Dopamine and Glutamate Concentrations in Rats
}

\author{
Hosseinali Ettehadi ${ }^{1}$, Seyedeh Nargesolsadat Mojabi ${ }^{2}$, Mina Ranjbaran ${ }^{2}$, \\ Jamal Shams $^{3}$, Hedayat Sahraei ${ }^{2 *}$, Mahdi Hedayati ${ }^{4}$, Farzad Asefi ${ }^{5}$ \\ ${ }^{1}$ Institute of Science and Technology, Tehran, Iran \\ ${ }^{2}$ Neuroscience Research Center, Baqyiatallah University of Medical Sciences, Tehran, Iran \\ ${ }^{3}$ Department of Psychiatry, Faculty of Medicine, and Neuroscience Research Center, \\ Shahid Beheshti University of Medical Sciences, Tehran, Iran \\ ${ }^{4}$ Fat Research Center, Shahid Beheshti University of Medical Sciences, Tehran, Iran \\ ${ }^{5}$ Department of Physiology, Faculty of Medicine, Shahid Beheshti University of Medical Sciences, Tehran, Iran \\ Email: *h.sahraei@bmsu.ac.ir
}

Received December 10, 2012; revised March 2, 2013; accepted March 20, 2013

Copyright (C) 2013 Hosseinali Ettehadi et al. This is an open access article distributed under the Creative Commons Attribution License, which permits unrestricted use, distribution, and reproduction in any medium, provided the original work is properly cited.

\begin{abstract}
Recent studies involving human and animal models have identified that saffron helps in the improvement of depression. Antidepressants are known to function in part by increasing brain serotonin, norepinephrine and dopamine concentrations. Therefore, to identify the cellular and molecular mechanism(s) underlying this property of saffron, we measured changes in rat brain dopamine, serotonin, norepinephrine and glutamate concentrations after administration of varying doses of an aqueous extract of saffron stigma. Male Wistar rats $(250 \pm 30 \mathrm{~g})$ were administered a single dose of saffron extract (5, 25, 50, 100, 150, and $250 \mathrm{mg} / \mathrm{kg}$, i.p.), fluoxetine (10 mg/kg, i.p.), and/or desipramine (50 mg/kg, i.p.) and were sacrificed $30 \mathrm{~min}$ later. Brains were removed, homogenized, and centrifuged at $4^{\circ} \mathrm{C}$. The supernatant was used for subsequent neurotransmitter detection by ELISA. Our results indicated that the aqueous extract of saffron (50, 100, 150 and $250 \mathrm{mg} / \mathrm{kg}$, i.p.) increased brain dopamine concentration in a dose-dependent manner compared with saline. In addition, the brain glutamate concentration increased in response to the highest dose of the extract $(250 \mathrm{mg} / \mathrm{kg}$, i.p.). Interestingly, the extract had no effect on brain serotonin or norepinephrine concentration. Our findings show that the aqueous extract of saffron contains an active component that can trigger production of important neurotransmitters in brain, namely, dopamine and glutamate. In addition, these results provide a cellular basis for reports concerning the antidepressant properties of saffron extract in humans and animals.
\end{abstract}

Keywords: Saffron; Glutamate; Dopamine; Norepinepherine; Serotonin

\section{Introduction}

Depression is a common and pernicious illness that can occur at many stages of life. Between $15 \%$ and $20 \%$ of patients have symptoms that persist for at least 2 years, and often these patients do not fully recover between depressive episodes [1]. Depression is also associated with high rates of relapse, recurrence, disability, and death [2]. Collectively, the high rates of chronicity, relapse, recurrence, morbidity and mortality highlight the importance of safe and effective long-term pharmacological treatment of this disease. However, evidence indicates that individuals with depression are seriously undertreated and receive inappropriate or inadequate treatment, with enor-

${ }^{*}$ Corresponding author. mous costs to individuals and society [1].

Saffron, Crocus sativus L. (Iridaceae), is used in folk medicine as an aphrodisiac and antispasmodic agent, expectorant and antidepressant [3]. Recent studies have demonstrated that saffron extract and its constituent, crocin, show interactions with morphine reward properties [4-10]. Interestingly, these studies have shown that the extract may interact with the neural elements located in the shell region of the nucleus accumbens [8]. On the other hand, Hosseinzadeh and Jahanian have shown that the extract and safranal and crocin can reduce the signs of morphine withdrawal syndrome in mice [11]. Chemical studies on Crocus sativus have shown the presence of constituents such as crocin, crocetin, safranal, and picrocrocin [12]. However, until today, there have been 
no studies on the effect of Crocus sativus extract on brain neurotransmitter concentrations. In the present study, the effect of saffron extract on brain serotonin, dopamine, norepinephrine and glutamate concentrations in male rats was investigated.

\section{Materias and Methods}

\subsection{Experimental Animals}

Male Wistar rats (250 - 300 g, Pasture Institute, Tehran, Iran) were used throughout the study (8 rats for each experiment). Animals were housed in groups of 4 /cage in a 12/12 h light-cycle (lights on at 07.00 a.m.), with ad-lib food and water available. The animals were randomly allocated to different groups of the experiment. All experiments were conducted in accordance with standard ethical guidelines and approved by the local ethical committee (The Baqiyatallah (a.s.) University of Medical Committee on the Use and Care of Animals, 81/021, July 10, 2002).

\subsection{Drugs}

Fluoxetine hydrochloride [N-methyl-3-[(4-trifluoromethyl) phenoxy]-3-phenylpropylamine hydrochloride] and desipramine hydrochloride [10-11-dihydro-N-methyl-5H-dibenz (Z) [b,f] azepine-5-propanamine hydrochloride] (TOCRIS Bioscience, UK) were dissolved in sterile saline and administered intraperitoneally at a concentration of $1 \mathrm{ml} / \mathrm{kg}$; the extract was prepared immediately before use. The control groups were administered saline.

\subsection{Plant Material}

The saffron used in this study was a gift from Talakaran-E-Mazraeh Agricultural Co. (Torbat Heydarieh, Khorasan-e-Razavi, Iran). The plant was authenticated by M. Kamalinejad (Department of Pharmacognosy, Faculty of Pharmacy, Shahid Beheshti University of Medical Sciences, Tehran, Iran) and a voucher specimen coded P-408 has been deposited at the herbarium of the Department of Pharmacognosy, Faculty of Pharmacy, Shahid Beheshti University of Medical Sciences, Tehran, Iran. Crocus sativus stigma is typically used as an additive and in herbal medicine. To prepare the extract, $100 \mathrm{~g}$ of dried and milled stigma was extracted with $1000 \mathrm{ml}$ distilled water by maceration. The extract was dried at $35^{\circ} \mathrm{C}-40^{\circ} \mathrm{C}$, and the yield of extraction was $23 \mathrm{mg}$ of freeze-dried powder per $100 \mathrm{mg}$ dry stigma. The extract was dissolved in normal saline and immediately administered to the animals.

\subsection{Brain Preparation}

Thirty minutes after drug and/or extract injection, animals were killed in a $\mathrm{CO}_{2}$ box [13], beheaded by a guil- lotine, and their brains were removed by a specialist in less than a minute. Brains were homogenized in a Falcon tube containing $10 \mathrm{ml}$ of cool $\left(0^{\circ} \mathrm{C}\right)$ sterile saline and centrifuged at $3000 \mathrm{rpm} / \mathrm{min}$ for $5 \mathrm{~min}$ at $4^{\circ} \mathrm{C}$. The supernatant was used for subsequent neurotransmitter detection by ELISA. On the basis of our previous studies, an interval time of $30 \mathrm{~min}$ was selected; this time was considered to be sufficient for extract action.

\subsection{Statistical Analysis}

Data are represent as means \pm standard error of mean (SEM) of the neurotransmitters concentration. One way analysis of variance (One-Way ANOVA) following by Tukey post hoc was used for statistical analysis. $P<0.05$ differences were considered significant.

\section{Results}

\subsection{Effects of Saffron Water Extract on Brain Serotonin Concentration}

The effect of different doses of saffron extract (5, 25, 50, 100, 150 and $200 \mathrm{mg} / \mathrm{kg}$, i.p.) on brain serotonin is shown in Figure 1. The animals received either saline (1 $\mathrm{ml} / \mathrm{kg}$, i.p.), or fluoxetine (10 $\mathrm{mg} / \mathrm{kg}$, i.p.), desipramine (50 mg/kg, i.p.), saffron extract (5, 25, 50, 100, 150 and $200 \mathrm{mg} / \mathrm{kg}$, i.p.) and $30 \mathrm{~min}$ later were sacrificed. One way ANOVA indicated that fluoxetine can increase brain serotonin significantly but neither desipramine nor saffron extract can increase brain serotonin $[F(8,64)=1.23$, $P<0.05]$, Figure 1.

\subsection{Effects of Saffron Extract on Brain Dopamine Level}

Our results indicated that both fluoxetine (10 mg/kg, i.p.) and desipramine (50 mg/kg, i.p.) can increase dopamine

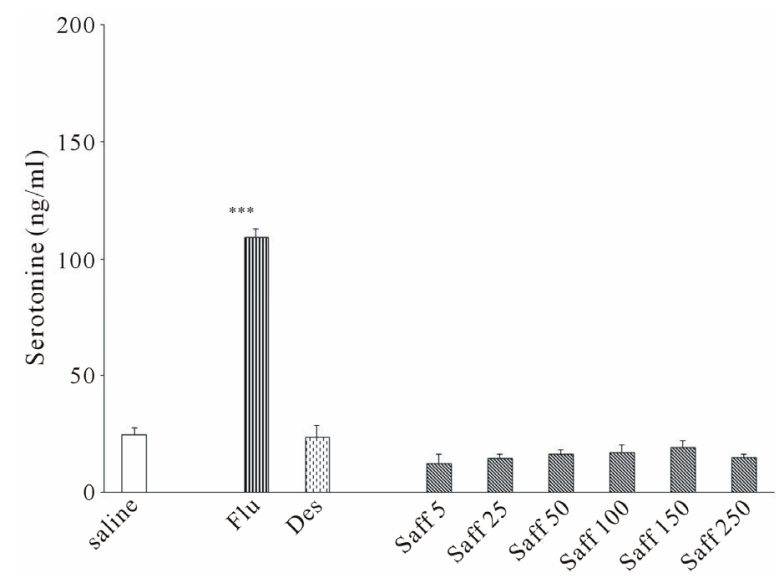

Figure 1. Effects of different doses of the aqueous extract of saffron on serotonin release in the brain. Each point is the mean \pm SEM of 8 rat brains. ${ }^{* * *} P<0.001$ different from saline control group. 
concentration on the animals. Interestingly, saffron extract can increase dopamine in a dose-dependent manner (Figure 2). Further analysis indicated that the extract dose of $250 \mathrm{mg} / \mathrm{kg}$, i.p. was more potent in this regard $[\mathrm{F}(8,64)=4.331, P<0.001]$, Figure 2 .

\subsection{The Effect of Saffron Extract on Brain Norepinephrine Concentration}

The effect of saffron extract on brain norepinephrine level is shown in Figure 3. Statistical analysis revealed that none of the drugs can increase the brain norepinephrine level $[\mathrm{F}(8,64)=0.79, P>0.05]$ (Figure 3), however, the fluctuations were observed in the responses which were not statistically significant.

\subsection{The Effect of Saffron Extract on Brain Glutamate Concentration}

In the last part of the experiments, the effect of saffron water extract on brain glutamate level was investigated. Our results indicated that there were fluctuations in brain glutamate level over doses of the extract. However, One way ANOVA revealed that the extract can increase the glutamate level in the dose of $250 \mathrm{mg} / \mathrm{kg}$, i.p. [F(8, 64) = 2.108, $P<0.01$ ], Figure 4 .

\section{Discussion}

Our studies have shown that the aqueous extract of saffron increases the release of important neurotransmitters, such as dopamine and glutamate in rat brains. This study also demonstrated that the effect of the extract on dopamine release was dose dependent. Since there is no study regarding the effect of saffron extract on neurotransmitter release, our results could not be compared with other investigations in this regard. However, it must be noted that in this study, the effect of the extract on other important neurotransmitters such as gamma-amino butyric acid (GABA) was not investigated. Moreover, it is not clear which specific constituents of the extract (e.g., safranal, crocin, and others) are responsible for the effects observed in our experiments. Finally, it must be noted that it was unclear whether dopamine or glutamate was still present in the vesicles or whether it was released into the synaptic space; this requires additional experiments to determine.

While our data show that the extract has no effect on serotonin and norepinephrine concentrations, it may be speculated that if the interval time between extract injection and brain removal was increased, changes in these neurotransmitter concentrations might be observed. This problem can be resolved by the explanation that in other experiments, the optimal time interval between extract injection and initiation of the extract effect was approximately $30 \mathrm{~min}[5,9,10]$. Several lines of evidence have

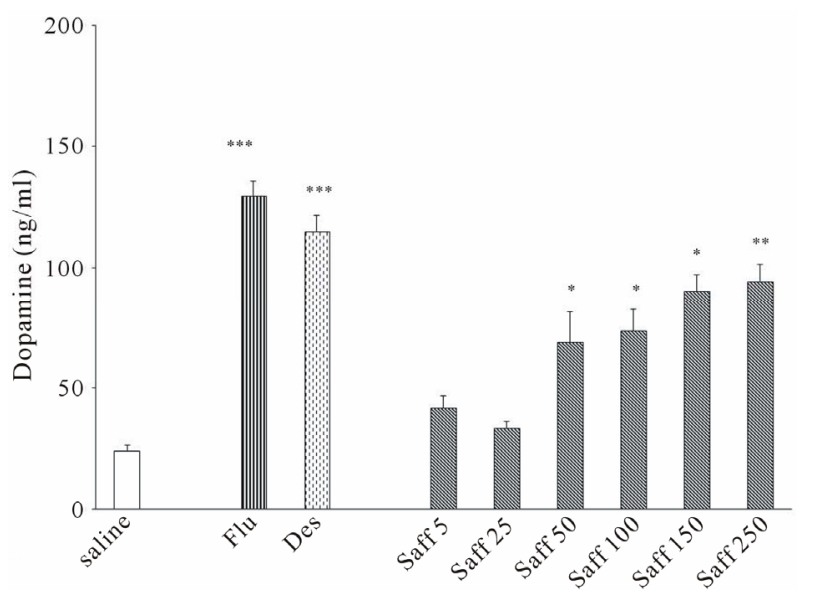

Figure 2. Effects of the aqueous extract of saffron on brain dopamine concentration. Each point is the mean \pm SEM of 8 rats. ${ }^{*} \boldsymbol{P}<0.05,{ }^{* *} \boldsymbol{P}<0.01$, and ${ }^{* * *} P<0.001$ vs the control group.

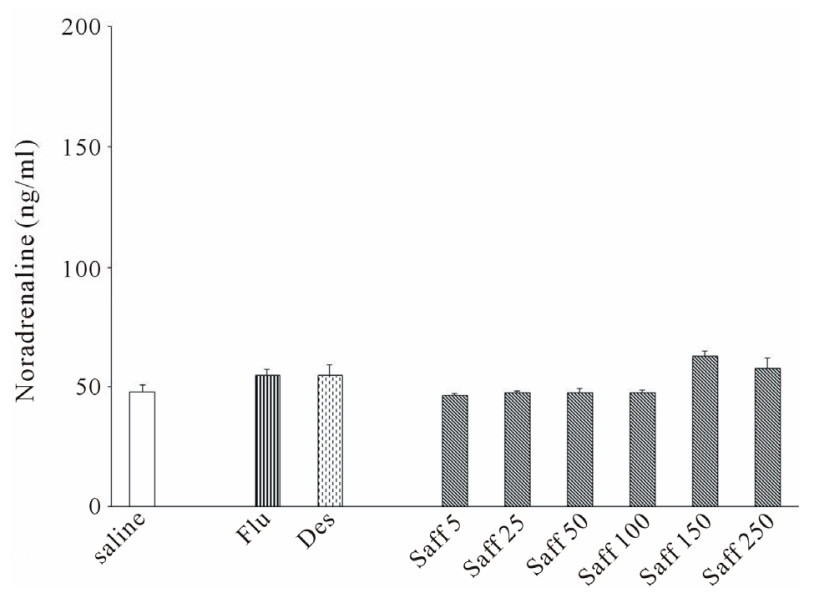

Figure 3. Effects of varying doses of the aqueous extract of saffron on brain noradrenaline concentration. Each point is the mean \pm SEM of 8 rat brains.

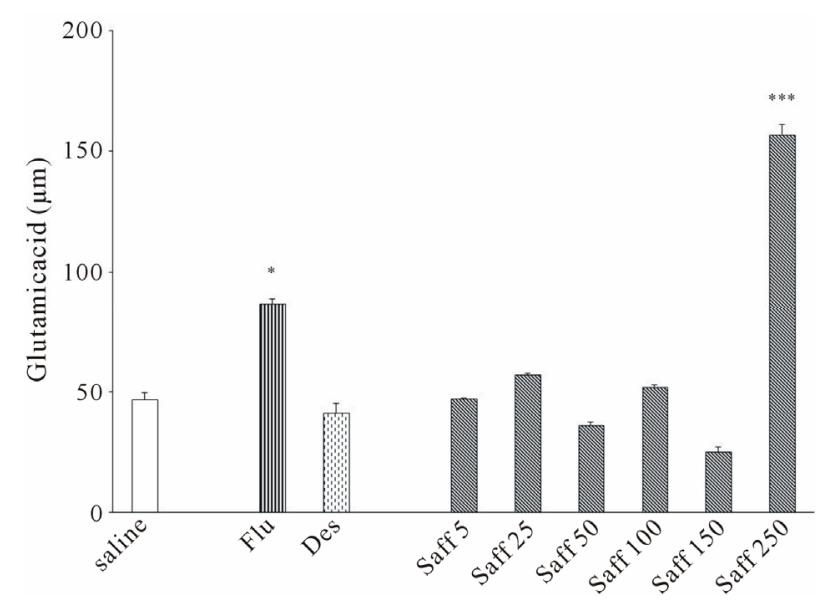

Figure 4. Effects of the aqueous extract of saffron on brain glutamate concentration. Each point is the mean \pm SEM of 8 rats. ${ }^{*} P<0.05$ and ${ }^{* * *} P<0.001$ vs the control group. 
indicated that both the aqueous and organic extracts of saffron can reduce depression in animals and humans [3, 13]. In this regard, it has shown that in comparison to imipramine, the ethanolic extract of saffron ( $30 \mathrm{mg} /$ day) can reduce signs of depression in patients [14]. In addition, the efficacy of the organic extract of saffron in mild-to-moderate depression improvement has been reported in another study $[15,16]$. Moreover, both the organic and aqueous extracts of saffron can reduce signs of depression induced by forced swimming stress in mice [17].

With regard to other mental disorders, investigators have shown that the organic extract of saffron can reduce signs of Alzheimer's disease during early phases of the disease [18]. Previous studies have also shown that the aqueous extract of saffron can induce rewarding properties, such as place preference and locomotor activity in both male and female mice $[9,10]$.

It is important to note that depression is defined by a serious reduction in brain monoamine concentration [1], and that antidepressant drugs increase monoamine concentration in the brain [1]. In this regard, investigators have shown that an increase in brain dopamine concentration, or administration of dopamine receptor agonists, may improve depression in humans and rats [19]. Our experiments have shown that brain dopamine concentration increases after treatment with saffron extract. Considering these two findings and other studies that saffron extract can improve depression; our results can be considered as being in accordance with those of previous studies. However, it is not clear which constituents of saffron extract are responsible for the observed increase in brain dopamine concentration. It must be noted that in previous studies, crocin has been identified as the most prominent constituent in the aqueous extract of saffron [12], and it has been shown to inhibit N-methyl-D-aspartate (NMDA) glutamate receptors and sigma opioid receptors isolated from rat spinal cord [20]. Moreover, as noted earlier, the extract can improve signs of Alzheimer's disease, probably because of its interaction with NMDA receptors [18]. Considering these facts, one can conclude that a constituent of the aqueous extract of saffron, possibly crocin, interacts with NMDA receptors in different parts of the brain to induce dopamine release. This model is in agreement with the ability of the extract to induce hyperactivity and place preference in mice $[5,9$, 10]. It is now clear that the dopamine concentration in the reward area induced by abused drugs is the cause of hyperactivity and place preference observed in animals $[21,22]$. Finally, the ability of the saffron extract to improve memory, inhibit neuronal degeneration, and reduce signs of depression may be because of the ability of the extract to induce dopamine and/or glutamate release.

It is important to note that in our experiments we did not measure regional changes in neurotransmitter levels in the central nervous system. For example, we did not focus on the change in dopamine concentration in brainspecific regions or serotonin concentration in the raphe nuclei. Thus, additional experiments are required to clarify this issue.

In this study, we injected the extract intraperitoneally, and it could be argued that different results might have been obtained if the extract was injected intravenously.

In conclusion: It found that the aqueous extract of saffron can induce dopamine and glutamate release in the brain, which we suggest is related to the effect of the extract on depression rehabilitation observed in previous studies.

\section{Acknowledgements}

This work was supported by the grants from Neuroscience Research Center, Baqiyatallah (a.s.) University of Medical Sciences and Neuroscience Research Center, Shahid Beheshti University of Medical Sciences.

\section{REFERENCES}

[1] J. J. Mann, "The Medical Management of Depression," The New England Journal of Medicine, Vol. 353, No. 17, 2005, pp. 1819-1834. doi:10.1056/NEJMra050730

[2] T. M. MacDonald, "Treatment of Depression: Prescription for Success?” Primary Care Psychiatry, Vol. 3, Suppl. 1, 1997, pp. 7-10.

[3] J. Sarris, "Herbal Medicines in the Treatment of Psychiatric Disorders: A Systematic Review,” Phytotherapy Research, Vol. 21, No. 8, 2007, pp. 703-716. doi:10.1002/ptr.2187

[4] M. Imenshahidi, H. Zafaria and H. Hosseinzadeh, "Effects of Crocin on the Acquisition and Reinstatement of Morphine-Induced Conditioned Place Preference in Mice,” Pharmacologyonline, Vol. 1, No. 1, 2011, pp. 10071013.

[5] B. Khakpour, M. Rostampour-Vajargah, H. Sahraei, M. Kamalinejad and J. Shams, "Effects of the Crocus sativus L. Extract on the Acquisition and Expression of Morphine-Induced Behavioral Sensitization in Male Mice," Kowsar Medical Journal, Vol. 12, No. 4, 2007, pp. 305313.

[6] M. Mobasher, H. Sahraei, B. Sadeghi-Rad, M. Kamalinejad and J. Shams, “ The Effects of the Crocus sativus Extract on the Acquisition and Expression of Morphine-Induced Conditioned Place Preference in Mice,” Journal of Rafsanjan University of Medical Sciences, Vol. 5, No. 3, 2005, pp. 143-150.

[7] N. Mojabi, A. Eidi, M. Kamalinejad, F. Khamseh, A. Khoshbaten, A. Noroozzadeh, F. Zighymat and H. Sahraei, "Study of the Effects of Alcoholic Extract of Crocus sativus on the Acquisition and Expression of Morphine-Induced Conditioned Place Preference in Rats," Kowsar Medical Journal, Vol. 13, No. 3, 2008a, pp. 197-210. 
[8] N. Mojabi, A. Eidi, M. Kamalinejad, F. Khamseh, A. Khoshbaten, A. Noroozzadeh, F. Zighymat and H. Sahraei, "Study of the Effects of Intra-Nucleus Accumbens Shell Injections of Alcohlic Extract of Crocus sativus on the Acquisition and Expression of Morphine-Induced Conditioned Place Preference in Rats," Physiology and Pharmacology, Vol. 12, No. 2, 2008b, pp. 121-128.

[9] H. Sahraei, J. Shams, S. Marjani, S. Molavi and M. Mohammadi, "Effects of the Crocus sativus L. Extract on the Acquisition and Expression of Morphine-Induced Behavioral Sensitization in Female Mice," Journal of Medicine Plants, Vol. 6, No. 21, 2007, pp. 26-35.

[10] H. Sahraei, M. Mohammadi, M. Kamalinejad, J. Shams, H. Ghoshooni and A. Noroozzadeh, "Effects of the Crocus sativus Extract on the Acquisition and Expression of Morphine-Induced Conditioned Place Preference in Female Mice,” Journal of Medicine Plants, Vol. 25, No. 1, 2005, pp. 40-49.

[11] H. Hosseinzadeh, and Z. Jahanian, "Effect of Crocus sativus L. (Saffron) Stigma and Its Constituents, Crocin and Safranal, on Morphine Withdrawal Syndrome in Mice,” Phytotherapy Research, Vol. 24, No. 5, 2010, pp. 726-30.

[12] M. Schmidt, G Betti and A. Hensel, "Saffron in Phytotherapy: Pharmacology and Clinical Uses,” Wiener Medizinische Wochenschrift, Vol. 157, No. 13-14, 2007, pp. 315-319. doi:10.1007/s10354-007-0428-4

[13] H. Zardooz, F. Rostamkhani, J. Zaringhalam and F. Faraji Shahrivar, "Plasma Corticosterone, Insulin and Glucose Changes Induced by Brief Exposure to Isoflurane, Diethyl Ether and $\mathrm{CO}_{2}$ in Male Rats," Physiolical Research, Vol. 59, No. 6, 2010, pp. 973-938.

[14] H. Hosseinzadeh and M. Nassiri-Asl, “Avicenna’s (Ibn Sina) the Canon of Medicine and Saffron (Crocus sativus): A review,” Phytotherapy Research, Vol. 27, No. 4, 2013, pp. 475-483. doi:10.1002/ptr.4784

[15] S. H. Akhondzadeh, H. Fallah-Pour, K. Afkham, A. H. Jamshidi and F. Khalighi-Cigaroudi, "Comparison of Crocus sativus L. and Imiperamine in the Treatment of Mild to Moderate Depression: A Pilot Double-Blind Ran- domized Trial," BMC Complementary and Alternative Medicine, Vol. 4, No. 1, 2004, pp. 12-16.

[16] A. A. Noorbala, S. H. Akhondzadeh, N. Tahmacebi-Pour and A. H. Jamshidi, "Hydro-Alcoholic Extract of Crocus sativus L. Versus Fluoxetine in the Treatment of Mild to Moderate Depression: A Double-Blind, Randomized Pilot Trial,” Journal of Ethnopharmacology, Vol. 97, No. 2, 2005, 281-284. doi:10.1016/j.jep.2004.11.004

[17] E. Moshiri, A. Noorbala, A. Jamshidi, S. H. Abbasi and S. H. Akhondzadeh, "Comparison of Petal of Crocus sativus L. and Fluoxetine in the Treatment of Depressed Outpatients: A Pilot Double-Blind Randomized Trial," Progress in Neuropsychopharmacology and Biological Psychiatry, Vol. 1, No. 2, 2007, pp. 439-442.

[18] G. Karimi, H. Hosseinzadeh and P. Khaleghpanah, "Study of Antidepressant Effect of Aqueous and Ethanolic of Crocus sativus in Mice," Iranian Journal of Basic Medical Sciences, Vol. 4, No. 3, 2001, pp. 11-15.

[19] S. Akhondzadeh, M. Shafiee-Sabet, M. H. Harirchian, M. Togha, H. Cheraghmakani, S. Razeghi, S. S. Hejazi, M. H. Yousefi, R. Alimardani, A. Jamshidi, F. Zare and A. Moradi, "Saffron in the Treatment of Patients with Mild to Moderate Alzheimer's Disease: A 16-Week, Randomized and Placebo-Controlled Trial," Journal of Clinical Pharmacy and Therapeutics, Vol. 35, No. 5, 2010, pp. 581588. doi:10.1111/j.1365-2710.2009.01133.x

[20] P. Dharmshaktu,V. Tayal and B. S. Kalra, "Efficacy of Antidepressants as Analgesics: A Review," The Journal of Clinical Pharmacology, Vol. 52, No. 1, 2012, pp. 6-17. doi:10.1177/0091270010394852

[21] M. Lechtenberg, D. Schepmann, M. Niehues, N. Hellenbrand, B. Wunsch and A. Hensel, "Quality and Functionality of Saffron: Quality Control, Species Assortment and Affinity of Extract and Isolated Saffron Compounds to NMDA and U1 (Sigma-1) Receptors," Planta Medica, Vol. 74, No. 7, 2008, pp. 764-772.

[22] J. Cami and M. Farre, "Drug Addiction,” The New England Journal of Medicine, Vol. 349, No. 10, 2003, pp. 975-986. doi:10.1056/NEJMra023160 